\title{
Role of the PEST sequence in the long-type GATA-6 DNA-binding protein expressed in human cancer cell lines
}

\author{
Kanako Obayashi ${ }^{1}$, Kayoko Takada ${ }^{1}$, Kazuaki Ohashi ${ }^{2}$, Ayako Kobayashi-Ohashi ${ }^{3}$, \\ Masatomo Maeda $^{4^{*}}$ \\ ${ }^{1}$ Laboratory of Biochemistry and Molecular Biology, Graduate School of Pharmaceutical Sciences, Osaka University, Suita, Japan \\ ${ }^{2}$ Department of Medical Biochemistry, School of Pharmacy, Iwate Medical University, Shiwa-Gun, Japan \\ ${ }^{3}$ Department of Immunobiology, School of Pharmacy, Iwate Medical University, Shiwa-Gun, Japan \\ ${ }^{4}$ Department of Molecular Biology, School of Pharmacy, Iwate Medical University, Shiwa-Gun, Japan \\ Email: ${ }^{*}$ mmaeda@iwate-med.ac.jp
}

Received 24 May 2012; revised 28 June 2012; accepted 4 July 2012

\begin{abstract}
GATA-6 mRNA utilizes two Met-codons in frame as translational initiation codons in cultured mammalian cells. Deletion of the nucleotide sequence encoding the PEST sequence between the two initiation codons unusually reduced the protein molecular size on SDS-polyacrylamide gel-electrophoresis. The reduced molecular size is ascribed to the molecular property of GATA-6, since both amino- and carboxylterminal tags introduced into GATA-6 were detected on the gel. This PEST sequence seems to contribute to expansion of the long-type GATA-6 molecule. The long-type GATA-6 containing the PEST sequence exhibits more activation potential than that without this sequence, the latter's activity being similar to that of the short-type GATA-6. We further demonstrated that human colon and lung cancer cell lines express both the long-type GATA-6 and the short-type GATA-6 in their nuclei.
\end{abstract}

Keywords: DNA-Binding Protein; GATA-6; Transcription Factor; Leaky Ribosome Scanning; PEST Sequence; Gel Electrophoresis

\section{INTRODUCTION}

Transcription factor GATA-6 contains tandem zinc fingers (CVNC-X17-CNAC)-X29-(CXNC-X17-CNAC) and recognizes a canonical DNA motif $(\mathrm{A} / \mathrm{T}) \operatorname{GATA}(\mathrm{A} / \mathrm{G})[1$, 2]. It regulates the expression of various genes required for developmental processes and tissue-specific functions [3]. Among mammalian GATA factors, GATA-6 is distinct in that it has a 146 extra-amino terminal extension compared with five other members $[3,4]$.

In vitro transfection of an expression plasmid for

${ }^{*}$ Corresponding author.
GATA-6 into cultured cells produced both long-type and short-type GATA-6, which are denoted as the L-type and S-type, respectively, from a single gene [4]. The same is true on in vitro translation of GATA-6 mRNA [5]. Sitedirected mutagenesis and deletion studies suggested that the translation of S-type GATA- 6 could be due to the leaky scanning of Met codons by ribosomes, but not the presence of an internal ribosome entry site in front of the coding region for S-type GATA-6 [4]. Furthermore, deletion of the protein sequence between Glu-31 and Cys-46, which is a typical PEST sequence closely related to protein degradation [6], in the L-type specific sequence reduced unusually the apparent molecular size [4].

To further study biological significance of the PEST sequence in the L-type GATA-6, molecular properties of L-type GATA-6 might be served more extensively. Actually, it has not been determined whether the correct amino- and carboxyl-terminal portions of GATA- 6 are present in the protein of the reduced size or not [4]. In this study we addressed this point by expressing human GATA-6 with human influenza hemagglutinin (HA)- and Myc-tags, and we suggest that the region between Glu31 and Cys-46 contributes to the unfolded structure of L-type GATA-6. We further demonstrated that the L-type GATA-6 is translated in established human cancer cell lines and is localized in their nuclei.

\section{MATERIALS AND METHODS}

\subsection{Cell Culture}

Cos-1 (ATCC) and A549 (RIKEN Cell Bank) cells were grown in Dulbecco's modified Eagle medium (GIBCO). CHO-K1 cells were cultured in Ham's F-12 medium (GIBCO). An expression plasmid was introduced into the cells by means of the diethylaminoethyl-dextran method, as described previously [4]. Cells were grown in $5 \mathrm{ml}$ of culture medium for $48 \mathrm{hrs}$ before harvesting. Protease 
inhibitors $[20 \mu \mathrm{M}$ benzyloxycarbonyl-Leu-Leu-norvalinal (MG115), $1 \mathrm{mM}$ phenylmethylsulfonyl fluoride (PMSF) and $50 \mu \mathrm{M}$ [(2S,3S)-3-Ethoxycarbonyloxirane-2-carbonyl]L-leucine (3-methylbutyl)amide (E-64d)] were added at $24 \mathrm{hr}$ before harvesting as a dimethyl sulfoxide solution $(10,25$ and $25 \mu 1 / 5 \mathrm{ml}$ medium, respectively). DLD-1 and HCT-15 (Cell Research Center for Biomedical Research, Tohoku University), and RKO (ATCC) cells were grown in RPMI-1640 medium (GIBCO). All the media were supplemented with $7 \%(\mathrm{v} / \mathrm{v})$ fetal bovine serum (JRH Biosciences).

\subsection{Construction of Expression Plasmds for GATA-6 with HA- and Myc-Tags}

To construct an expression plasmid for L-type GATA-6 with an amino-terminal HA-tag, phosphorylated doublestranded oligonucleotides (KO003/KO004 and KO005/ KO006 pairs, Table 1) were inserted between the XhoI and EcoRV sites of pBluescript II SK(+). The XhoI NheI fragment of the resulting plasmid was replaced with the corresponding fragment of pME-hGT1L5'uL or pMEhGT1L5' $\triangle \mathrm{EuL}$ [4]. The constructs were named pMEhGT1LHA and pME-hGT1L $\triangle$ EHA, respectively (Figure 1(a)).

To introduce an Myc-tag to the carboxyl-terminus of
L-type GATA-6 with an amino-terminal HA-tag, synthetic DNA encoding an Myc-tag (KO013/KO014) was inserted between the unique AvrII and SpeI sites of pBluescript-KaeI encoding a fusion protein of S-type GATA-6 and carboxyl-terminal half of SREBP-2 [7]. The AccI-SpeI fragment was substituted with pMEhGT1LHA or pME-hGT1LAEHA to construct pME-hGT1LHAMyc and pME-hGT1L $\Delta$ EHA-Myc, respectively (Figure 1(b)). The DNA sequence was confirmed by the dideoxy chaintermination method [8] using sequencing primers listed in the Table 1. The molecular biological techniques were performed by the published methods [9].

\subsection{SDS-Polyacrylamide Gel-Electrophoresis and Western Blotting}

A nuclear extract (10 $\mu \mathrm{g}$ protein) of transfected cells [4] was subjected to sodium dodecyl sulfate (SDS)-polyacrylamide gel $[7.5 \%$ or $10 \%(\mathrm{w} / \mathrm{v}), 1 \mathrm{~mm}$ thickness] electrophoresis [10], and then electro-blotted $(200 \mathrm{~mA}$, 90 min, ATTO Model-AE6675) onto an Immobilon ${ }^{\text {TM }}$-P membrane [Millipore PVDF membrane $(0.45 \mu \mathrm{m})$, IPVH00010]. The filter was blocked overnight at $4^{\circ} \mathrm{C}$ with $10 \mathrm{mM}$ sodium phosphate buffer (pH 7.2), $137 \mathrm{mM}$ $\mathrm{NaCl}, 3 \mathrm{mM} \mathrm{KCl}$ containing $0.1 \%$ (v/v) Tween 20 and $3 \%(\mathrm{w} / \mathrm{v})$ bovine serum albumin (Wako). Rabbit site-

Table 1. Oligonucleotides used for cassette mutagenesis and sequencing.

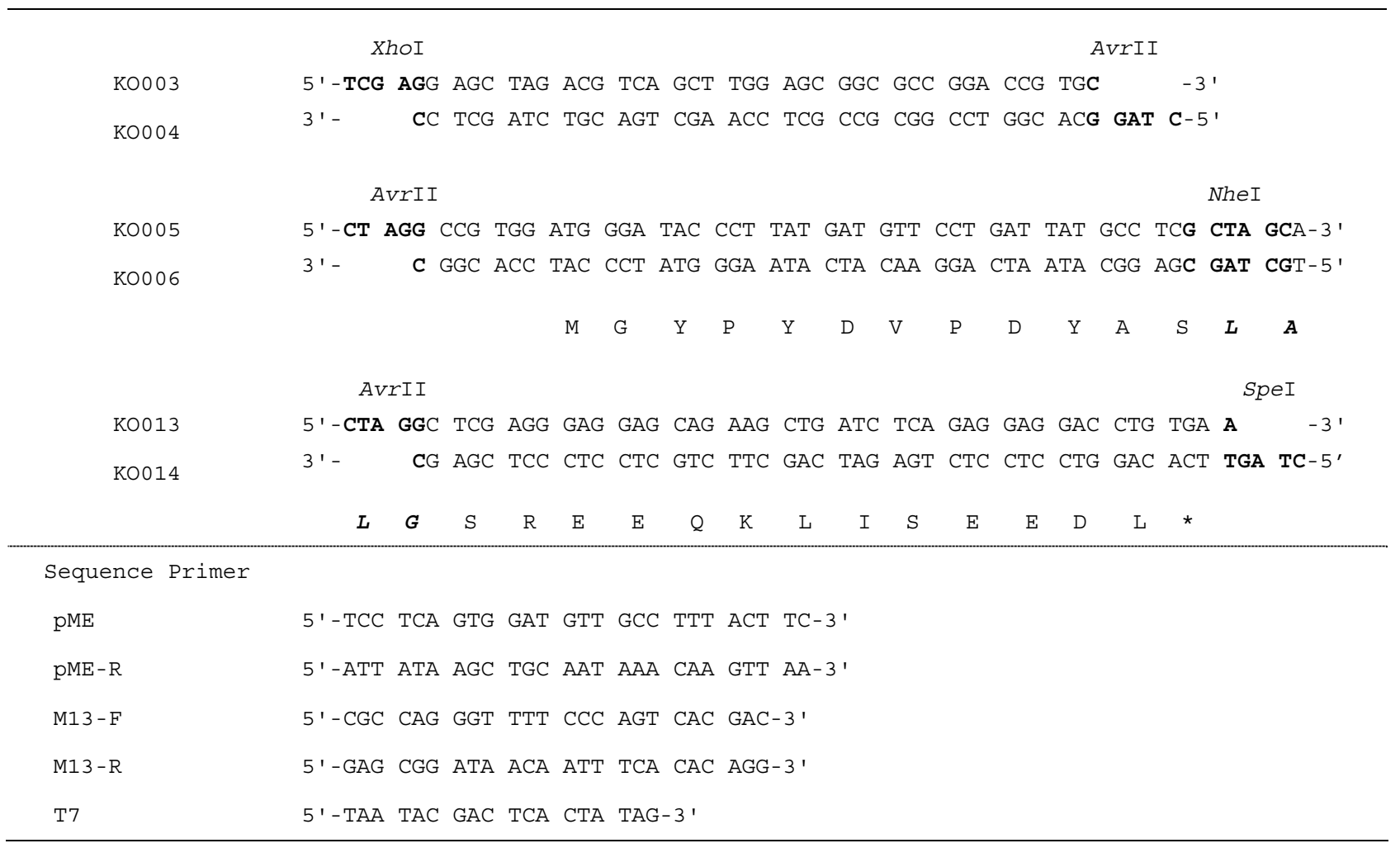

Bold letters indicate the restriction enzyme sites. Amino acid sequences for HA- and Myc-tags are shown under the second and third cassette sequences, respectively. Bold italic letters indicate the dipeptide linker sequence between GATA-6 and Myc-tag. 


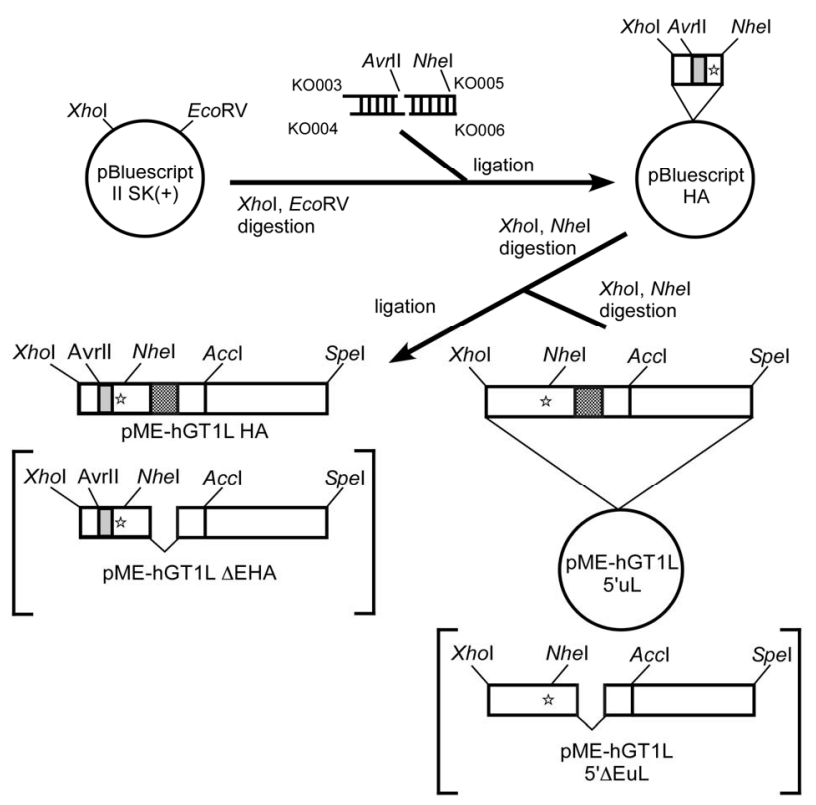

(a)

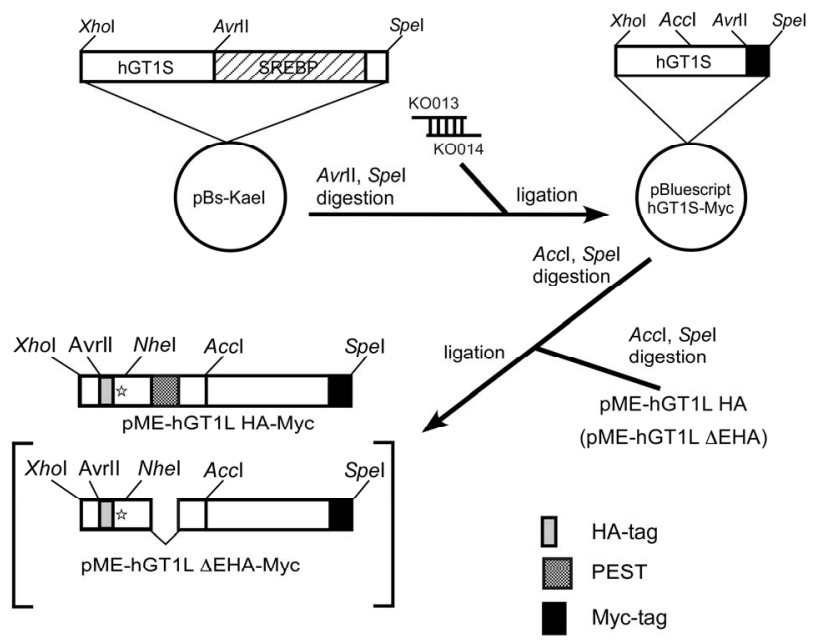

(b)

Figure 1. Construction of expression plasmids for GATA-6 with HA- and Myc-tags. Expression plasmids for L-type GATA-6 with an amino-terminal HA-tag (pME-hGT1LHA and pMEhGT1LAEHA) were constructed as described in Materials and Methods using phosphorylated double-stranded oligonucleotides (Table 1) and pME-hGT1L5'uL or pME-hGT1L5' $\Delta$ EuL (a). An Myc-tag was introduced to the carboxyl-terminus of L-type GATA-6 with an amino-terminal HA-tag (pME-hGT1LHA-Myc and pME-hGT1L $\Delta$ EHA-Myc) (b).

specific polyclonal antibody GATA-6N recognizing human S-type GATA-6 (Leu59 - Gln217) [6] was used as the first antibody $(\times 500$ diluted $)$. Horseradish peroxidaselinked donkey anti-rabbit immunoglobulin $(\times 4000$ diluted) (GE Healthcare) was used as the second antibody. Chemiluminescence was detected with a Western blotting kit (GE Healthcare) using Scientific Imaging Film (KODAK).
The HA-tag was detected with HA-7 $(\times 10,000$ diluted $)$ (Sigma), followed by horseradish peroxidase-linked antimouse IgG (GE Healthcare) $(\times 4000$ diluted) as the second antibody. The Myc-tag reacted with the peroxidaselinked mouse anti-c-Myc antibody (MC045, Nacalai Tesque) $(\times 1500$ diluted). Reprobing was carried out as follows. The membrane was treated with buffer $[2 \%(\mathrm{w} / \mathrm{v})$ SDS, $100 \mathrm{mM} \beta$-mercaptoethanol, $62.5 \mathrm{mM}$ Tris- $\mathrm{HCl}$ (pH 6.7)] for $30 \mathrm{~min}$ at $50^{\circ} \mathrm{C}$, blocked overnight at $4^{\circ} \mathrm{C}$, and then reacted with the antibody in the same way as for GATA-6N. The amino acid residue numbers were based on the sequence of the S-type GATA-6 [6]. Protein concentrations were determined with a BCA Protein Assay (Pierce) using bovine serum albumin (Fraction V, Sigma) as a standard [11].

\subsection{Immunoprecipitation of GATA-6 from Nuclear Extracts of Various Human Cancer Cells}

All the procedures were carried out at $4^{\circ} \mathrm{C}$. Cells $/ \Phi 10 \mathrm{~cm}$ dish were collected in a $1 \mathrm{ml}$ of ice-cold $20 \mathrm{mM}$ Tris- $\mathrm{HCl}$ ( $\mathrm{pH} 7.5), 150 \mathrm{mM} \mathrm{NaCl}, 2 \mathrm{mM}$ EDTA, $10 \mu \mathrm{g} / \mu \mathrm{l}$ leupeptin, $10 \mu \mathrm{g} / \mu \mathrm{l}$ pepstatin A (TNE buffer) containing $1 \%$ (w/v) NP-40 and kept on ice for $30 \mathrm{~min}$. After sheering the suspension 5 - 10 times through a $25 \mathrm{G}$ needle, a supernatant $(12,000 \times \mathrm{g}, 30 \mathrm{~min})$ was obtained. Protein $\mathrm{G}$ Sepharose beads (GE Healthcare) were pre-washed with TNE buffer containing NP-40. An aliquot of the supernatant $(0.8 \mathrm{mg}$ protein in $400 \mu \mathrm{l})$ was incubated with Protein G Sepharose beads ( $40 \mu \mathrm{l}$ bed volume) for $1 \mathrm{hr}$ in a Mini Disk Rotor BC-710 (BIO CRAFT) [12], and then centrifuged $(2000 \times \mathrm{g}, 5 \mathrm{~min})$. The Protein G-treated supernatant was reacted with anti-GATA-6 (C20; Santa Cruz) for $1 \mathrm{hr}$ in the rotor. The prewashed Protein G Sepharose beads ( $40 \mu \mathrm{l}$ bed volume) were added, followed by incubation for $1 \mathrm{hr}$. The beads were precipitated $(2000 \times \mathrm{g}, 5 \mathrm{~min})$ and then washed five times with $200 \mu \mathrm{l}$ of TNE buffer without NP-40 and protease inhibitors. The recovered immunocomplex was heated at $95^{\circ} \mathrm{C}$ for $5 \mathrm{~min}$ after the addition of $10 \mu \mathrm{l}$ of $2 \times$ sample buffer [10]. The solubilized protein was subjected to SDSpolyacrylamide gel-electrophoresis, and then to Western blotting with the GATA-6N antibody.

\subsection{Reporter Gene Assay}

In each well of a 6 -well culture plate, $1 \times 10^{5} \mathrm{CHO}-\mathrm{K} 1$ cells were seeded into $2 \mathrm{ml}$ Ham's F12 medium containing $7 \%(\mathrm{v} / \mathrm{v})$ fetal bovine serum, and then cultured for 24 hr. Lipofectamine ${ }^{\mathrm{TM}}$ (Invitrogen) was used for transfection (duplicate) of plasmid DNA mix, reporter plasmid p8GATA/GL3 [4] carrying a hybrid promoter with three tandem $\mathrm{R} \beta_{2}$ segments of rat $\mathrm{H}^{+} / \mathrm{K}^{+}$-ATPase $\beta$ subunit gene promoter [13] and the short segment of rat intrinsic 
factor gene promoter [14] $(1 \mu \mathrm{g})$, GATA-6 expression plasmid $(0.6 \mu \mathrm{g}$ of pME-hGT1S, pME-hGT1LK, pMEhGT1LEK or pME18S), and pSV- $\beta$-Gal $(0.5 \mu \mathrm{g})$ per well as described previously [4]. Lysis buffer $(90 \mu \mathrm{l})$ was added at $53 \mathrm{hrs}$ after the start of transfection, and a cell lysate was prepared $\left(12,000 \times \mathrm{g}, 10 \mathrm{~min}\right.$ at $\left.4^{\circ} \mathrm{C}\right)$. An aliquot $(20 \mu \mathrm{l}$ and $5 \mu \mathrm{l})$ of the supernatant was used for measurement of the luciferase and $\beta$-galactosidase activties, respectively [4]. The activity was normalized as to the $\beta$-galactosidase activity.

\subsection{Chemicals}

Restriction enzymes were purchased from NEB and Toyobo. T4 DNA ligase and Agarose-LE Classic Type were supplied by TaKaRa. T4 polynucleotide kinase and calf intestine phosphatase were obtained from NEB. Ampli Taq was from Roche. Oligonucleotides were purchased from Invitrogen. Leupeptin, pepstatin A and PMSF were provided by Sigma. MG115 and E-64d were from the Peptide Institute. All other chemicals used were of the highest grade commercially available.

\section{RESULTS}

\subsection{Detection of Amino- and Carboxyl-Termini of the L-Type GATA-6 of Apparent Reduced Size}

In our previous study [4], deletion of the PEST sequence ( $\triangle \mathrm{EX}$ with 506 residues) unusually reduced the protein molecular size on SDS-polyacrylamide gel-electrophoresis compared with the other deletion with similar amino acid residue number $(\triangle \mathrm{SB}$ with 507 residues carrying the PEST sequence). To examine whether the apparent increased mobility of the $\Delta \mathrm{EX}$ recombinant protein could be due to proteolytic processing or not, we added amino- and carboxyl-terminal tags.

Both the L-type and S-type GATA-6 proteins with an amino-terminal HA-tag produced from pME-hGT1LHA and pME-hGT1LAEHA (Figure 2(a), lanes 4 and 5) were essentially the same size as those produced from pME-hGT1L and pME-hGT1L $\Delta$ EX, respectively, (Figure 2(a), lanes 2 and 3), as determined with polyclonal antibodies for S-type GATA-6. Furthermore, the L-type GATA-6 with or without the PEST sequence has an amino-terminus, since a HA-tag was detectable (Figure 2(b), lanes 4 and 5). The S-type GATA-6 detected in Figure 2(a) (lanes 4 and 5) did not react with antibodies to a HA-tag since it was produced through leaky ribosome scanning (Figure 2(d)) [4].

When the L-type with an amino-terminal HA-tag and a carboxyl-terminal Myc-tag was expressed from pMEhGT1LHA-Myc or pME-hGT1LAEHA-Myc, the molecular sizes of both the L-type and S-type GATA-6 slightly (a)

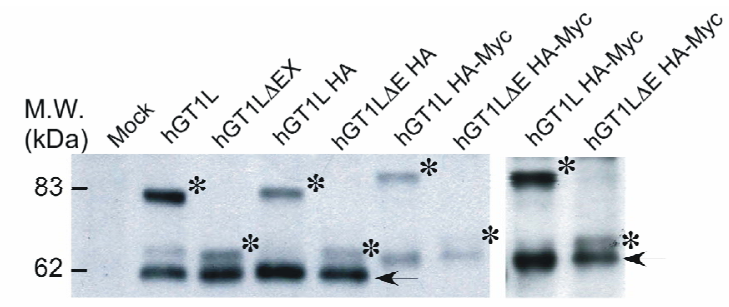

(b)

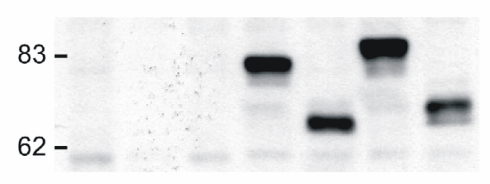

(c)

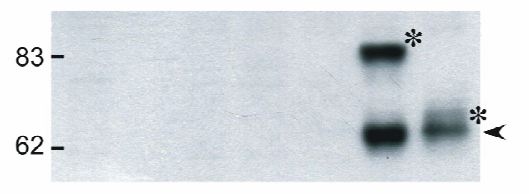

(d)

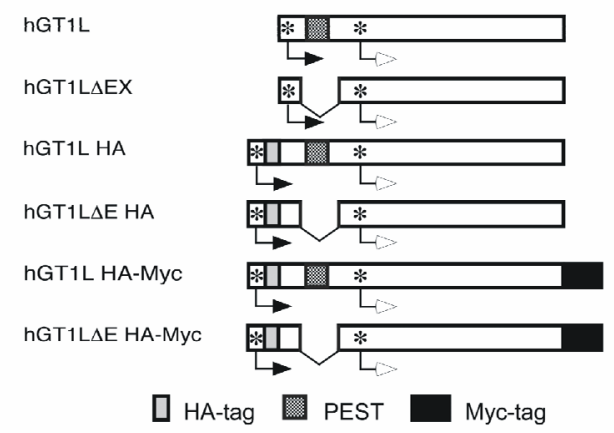

Figure 2. Detection of the amino- and carboxyl-termini of GATA- 6 of reduced size. Cos-1 cells were transfected with pME-hGT1L, pME-hGT1LAEX, pME-hGT1LHA, pMEhGT1LAEHA, pME-hGT1LHA-Myc, pME-hGT1L $\Delta$ EHA-Myc or pME18S (mock transfection). After two days, nuclear extracts were prepared, then analyzed by Western-blotting with antibodies for hGATA-6N (a), HA (b), and Myc (c). The positions of the L-type GATA-6 with or without the PEST sequence are indicated by asterisks, and those of the S-type GATA- 6 are indicated by arrows in (a) and (c). Lanes 6 and 7 in (a) were exposed extensively and are shown at the right of panel (a). The expression plasmids introduced are schematically shown in (d). The closed arrows indicate the translation of L-type GATA-6, while the open arrows indicate that of S-type GATA-6 through leaky ribosome scanning [4]. The dotted, grey-colored and closed boxes indicate the PEST sequence, HA-tag and Myc-tag, respectively.

increased (Figure 2(a), lanes 6 and 7). The L-type with or without the PEST sequence had a HA-tag while the S-type did not (Figure 2(b), lanes 6 and 7). Both L-type and S-type GATA-6 had an Myc-tag, as detected with the antibodies for an Myc-tag (Figure 2(c), lanes 6 and 7). From these results it is evident that the L-type with or without PEST sequence had both amino- and carboxyltermini, and that the decrease in the molecular weight of the L-type is due to a structural reason, but not to proteolytic degradation. 


\subsection{Transcriptional Activation Competency of L-Type GATA-6 with or without the PEST Sequence in the Reporter Gene Assay}

A plasmid construct with the Kozak sequence around the initiator Met-codon for L-type GATA-6 (pME-hGT1L5'K and pME-hGT1L5' $\Delta \mathrm{EK}$ ) produced only the L-type [4]. Then we examined whether the L-type GATA- 6 with or without the PEST sequence functions differently in terms of transcriptional activation of the GATA-responsive reporter gene [4,15]. As shown in Figure 3, the expression of the L-type from pME-hGT1L5'K showed strong activation of the reporter gene compared with that of the S-type from pME-hGT1S, essentially similar to as previously reported [4]. Interestingly, the expression of the L-type without the PEST sequence from pME-hGT1L5' $\triangle \mathrm{EK}$ did not activate the reporter gene significantly, the activation level being comparable to that in the case of the S-type. Thus, the PEST sequence positively affects the transcriptional activity of GATA-6.

\subsection{Detection of L-Type GATA-6 in Cultured Cells}

Although L-type GATA-6 is reproducibly detectable in a transiently expression system, it is important to demonstrate the native L-type in tissues and cultured cells. So

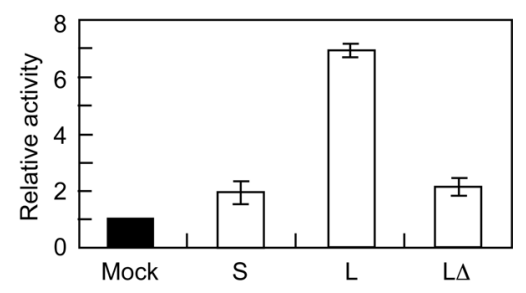

Figure 3. Transcriptional activities of Ltype GATA-6 with or without the PEST sequence, and S-type GATA-6. Expression plasmids for L- and S-type GATA-6 (pME-hGT1L5'K and pME-hGT1L5' $\Delta$ EK, and pME-hGT1S, respectively) were introduced into $\mathrm{CHO}-\mathrm{K} 1$ cells together with a reporter gene plasmid (p8GATA/ GL3) plus a $\beta$-galactosidase expression plasmid [4]. Luciferase activities were normalized as to those of the $\beta$-galactosidase. The relative activities as to mock transfection (pME18S instead of expression plasmids for GATA-6) are shown with the deviation for two independent experiments. The average value for $\mathrm{pME}$ 18S (Mock) was $9.5( \pm 0.1) \times 10^{5}(\mathrm{RLU} /$ $\mathrm{mU})$. S, S-type GATA-6 expression from pME-hGT1S; L, full-length L-type expression from pME-hGT1L5' $\mathrm{K} ; \Delta \mathrm{L}$, expression of the L-type without the PEST sequence from pME-hGT1L5' $\triangle \mathrm{EK}$. we evaluated the GATA- 6 expression in human cancer cells (cololectal DLD-1, RKO and Hct 15, and lung A549). It must be noted that S-type GATA-6 was detected in DLD-1 and RKO cells [16], and the GATA-6 gene was transcribed in A549 cells [17]. So we examined whether the L-type GATA-6 is expressed in these cells or not. A nuclear extract of these cells was immuno-precipitated with antibodies recognizing carboxyl-terminal GATA- 6 and detected by Western blotting with antibodies for amino terminal GATA-6.

As shown in Figure 4, L-type GATA-6 was present in all cells examined (lanes 3 - 6), and was indistinguishable from the L-type protein transiently expressed from pME-hGT1L in Cos-1 cells (lane 1). When only L-type GATA-6 was expressed from pME-hGT1L5' $\mathrm{K}$ in Cos-1 cells and was immuno-precipitated, a similar band to that of L-type GATA-6 was detected (lane 2). A corresponding band was not detected without a nuclear extract (lane 7). The S-type GATA-6 (denoted by white arrow heads in lanes $3-6$ ) was overlapped in the intense bands (denoted by brackets in lanes 2 and 7). Thus, the role of GATA- 6 in gene regulation must be carefully studied considering L-type GATA-6.

\section{DISCUSSION}

Deletion of amino acid residues Glu-31 - Cys-46 that con-

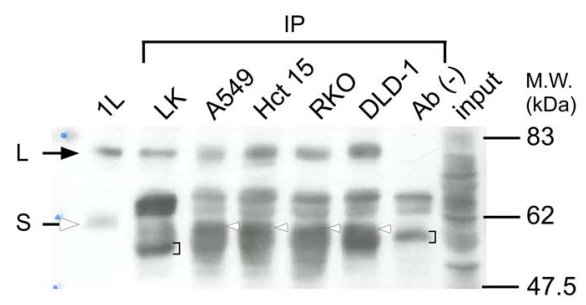

Figure 4. Detection of L-type GATA-6 in human cancer cell lines. Nuclear extracts were prepared from A549, Hct 15, RKO and DLD-1 cells, and then incubated with GATA-6 (C20) antibodies, followed by Protein G beads treatment. The bound proteins were subjected to SDS-polyacrylamide gel-electrophoresis and Western blotting. GATA-6 was detected with hGATA-6N antibodies (lanes 3 - 6). L-type GATA-6 was similarly recovered from a nuclear extract of Cos-1 cells transfected with pME-hGT1L5' K only expressing L-type GATA6 [4] (lane 2). A nuclear extract of Cos-1 cells transfected with pME-hGT1L shows the positions of L and S-type GATA-6 (lane 1, closed and open arrows, respectively). A nuclear extract of DLD-1 without any treatment (lane 8) and the Protein $\mathrm{G}$ beads directly treated with the SDS sample buffer (lane 7) were also analyzed. Open arrows and blackest indicate S-type GATA-6 and non-specific bands, respectively. 
stitute the PEST sequence together with the neighboring Arg residues (Arg-30 and Arg-48) [6] decreased the apparent molecular weight of L-type GATA-6 on SDSpolyacrylamide gel-electrophoresis. Re-introduction of the correct amino acid sequence restored its molecular size, while an unrelated sequence did not [4]. Such a reduction in molecular size could not be ascribed to partial digestion of the L-type GATA-6, since it had aminoand carboxyl-termini (Figure 2).

Proline-rich insect viral protein is known to exhibit abnormally low mobility on SDS-polyacrylamide gelelectrophoresis [18]. Furthermore, a carboxyl-terminal fragment of bacterial $\sigma^{70}$-factor with the Trp $\rightarrow$ Gly mutation has a decreased helical content, resulting in slower mobility on an SDS-gel [19]. Thus, the L-type GATA-6 with the PEST sequence is unpacked, especially in the amino-terminal region. Such a structure would retard its mobility on a gel. It must be further mentioned that the present study suggests additional role of the PEST sequence other than protein degradation signal [6].

It has been reported that a Pro-rich sequence often interacts with other proteins; DNA damage specifically induces p53 phosphorylation of Ser/Thr-Pro motifs, facilitating their interaction with peptidyl-prolyl isomerase, which stimulates the DNA-binding and the transactivation function of p53 [20]. Furthermore, the proline repeat (PXXP) domain of p53 binds directly to transcriptional coactivator p300 and the DNA-bound p53 is susceptible to acetylation by associated p300 [21]. The PXXP and PPXY motifs of the p63 variant are also required for the transcriptional activity [22]. It would be of interest to determine whether or not the Pro-rich half of the PEST sequence in L-type GATA-6 interacts with other regulatory protein(s), since the activation potential of L-type GATA-6 with the PEST sequence is higher than that without the PEST sequence.

In this study, we also found the expression of L-type GATA-6 in human colon and lung cancer cells. Furthermore, the L-type is exclusively detected in tissues such as human adrenal [23], and mouse embryonic heart myocardium, gut, pulmonary system and chondrogenesis sites [24]. It is suggested that GATA-6 plays important roles in regulation of the cell cycle [25] and apoptosis [16]. Furthermore, GATA-6 expression is interested in from the viewpoint of controlling the differentiation or maintenance of embryonic stem cells in vitro [26]. Thus, studies focused on both L- and S-type GATA-6 will provide valuable information in the relevant fields, as the presence of L-type GATA-6 has not been considered.

\section{ACKNOWLEDGEMENTS}

This research was supported in part by grants from MEXT [Grant-inAids for Scientific Research B (14370744) to M.M. and for Strategic
Medical Science Research Centers, 2010-2014 (The MIAST Project)].

\section{REFERENCES}

[1] Maeda, M., Kubo, K., Nishi, T. and Futai, M. (1996) Roles of gastric GATA DNA-binding proteins. Journal of Experimental Biology, 199, 513-520.

[2] Molkentin, J.D. (2000) The zinc finger-containing transcription factors GATA-4, -5 , and -6; ubiquitously expressed regulators of tissue-specific gene expression. Journal of Biological Chemistry, 275, 38949-38952. doi:10.1074/jbc.R000029200

[3] Maeda, M., Ohashi, K. and Ohashi-Kobayashi, A. (2005) Further extension of mammalian GATA-6. Development, Growth and Differentiation, 47, 591-600. doi:10.1111/j.1440-169X.2005.00837.x

[4] Takeda, M., Obayashi, K., Kobayashi, A. and Maeda, M. (2004) A unique role of an amino terminal 16-residue region of long-type GATA-6. Journal of Biochemistry, 135, 639-650. doi:10.1093/jb/mvh077

[5] Brewer, A., Gove, C., Davies, A., McNulty, C., Barrow, D., Koutsourakisi, M., Farzaneh, F., Pizzey, J., Bomford, A. and Patient, R. (1999) The human and mouse GATA-6 genes utilize two promoters and two initiation codons. Journal of Biological Chemistry, 274, 38004-38016. doi:10.1074/jbc.274.53.38004

[6] Rogers, S., Wells, R. and Rechsteiner, M. (1986) Amino acid sequence common to rapidly degraded proteins: The PEST hypothesis. Science, 234, 364-368. doi:10.1126/science. 2876518

[7] Tsuge, T., Uetani, K., Sato, R., Ohashi-Kobayashi, A. and Maeda, M. (2008) Cyclic AMP-dependent proteolysis of GATA-6 expressed on the intracellular membrane. Cell Biology International, 32, 298-303. doi:10.1016/j.cellbi.2007.10.005

[8] Sanger, F., Coulson, A.R., Barrell, B.G., Smith, A.J.H. and Roe, B.A. (1980) Cloning in single-stranded bacteriophage as an aid to rapid DNA sequencing. Journal of Molecular Biology, 143, 161-178. doi:10.1016/0022-2836(80)90196-5

[9] Sambrook, J., Fritsch, E.F. and Maniatis, T. (1989) Molecular cloning: A laboratory manual. 2nd Edition, Cold Spring Harbor Laboratory, Cold Spring Harbor.

[10] Laemmli, U.K. (1970) Cleavage of structural proteins during the assembly of the bacteriophage T4. Nature, 227, 680-685. doi:10.1038/227680a0

[11] Smith, P.K., Krohn, R.I., Hermanson, G.T., Mallia, A.K., Gartner, F.H., Provenzano, M.D., Fujimoto, E.K., Goeke, N.M., Olson, B.J. and Klenk, D.C. (1985) Measurement of proteins using bicinchroninic acid. Analytical Biochemistry, 150, 76-85. doi:10.1016/0003-2697(85)90442-7

[12] Arimochi, J., Ohashi-Kobayashi, A. and Maeda, M. (2007) Interaction of Mat-8 (FXYD-3) with $\mathrm{Na}^{+} / \mathrm{K}^{+}$-ATPase in colorectal cancer cells. Biological and Pharmaceutical Bulletin, 30, 648-654. doi:10.1248/bpb.30.648

[13] Maeda, M., Oshiman, K., Tamura, S., Kaya, S., Mahmood, S., Reuben, M.A., Lasater, L.S., Sachs, G. and Fu- 
tai, M. (1991) The rat $\mathrm{H}^{+} / \mathrm{K}^{+}$-ATPase subunit gene and recognition of its control region by gastric DNA binding protein. Journal of Biological Chemistry, 266, 2158421588.

[14] Maeda, M., Asahara, S., Nishi, T., Mushiake, S., Oka, T., Shimada, S., Chiba, T., Tohyama, M. and Futai, M. (1995) The rat intrinsic factor gene: Its 5'-upstream region and chief cell-specific transcription. Journal of Biochemistry, 117, 1305-1311.

[15] Nishi, T., Kubo, K., Hasebe, M., Maeda, M. and Futai, M. (1997) Transcriptional activation of $\mathrm{H}^{+} / \mathrm{K}^{+}$-ATPase genes by gastric GATA binding proteins. Journal of Biochemistry, 121, 922-929.

doi:10.1093/oxfordjournals.jbchem.a021674

[16] Shureiqi, I., Jiang, W., Fischer, S.M., Xu, X., Chen, D., Lee, J.J., Lotan, R. and Lippman, S.M. (2002) GATA-6 transcriptional regulation of 15-lipoxygenase-1 during NSAID-induced apotosis in colorectal cancer cells. Cancer Research, 62, 1178-1183.

[17] Guo, M., Akiyama, Y., House, M.G., Hooker, C.M., Heath, E., Gabrielson, E., Yang, S.C., Han, Y., Baylin, S.B., Herman, J.G. and Brock, M.V. (2004) Hypermethylation of the GATA genes in lung cancer. Clinical Cancer Research, 10, 7917-7924. doi:10.1158/1078-0432.CCR-04-1140

[18] Pham, D.Q.-D. and Sivasubramanian, N. (1992) Sequence and in vitro translational analysis of a 1629-nucleotide ORF in Autographa californica nuclear polyhedrosis virus strain E2. Gene, 122, 345-348. doi:10.1016/0378-1119(92)90224-D

[19] Gopal, V., Ma, H.-W., Kumara, M.K. and Chatterji, D. (1994) A point mutation at the junction of domain 2.3/2.4 of transcription factor $\sigma^{70}$ abrogates productive transcripttion and restores its expected mobility on a denaturing gel. Journal of Molecular Biology, 242, 9-22. doi:10.1006/jmbi.1994.1553

[20] Zheng, H., You, H., Zhou, X.Z., Murray, S.A., Uchida, T.,
Wulf, G., Gu, L., Tang, X., Lu, K.P. and Xiao, Z.-X.J. (2002) The prolyl isomerase Pin1 is a regulator of p53 in genotoxic response. Nature, 419, 849-857. doi:10.1038/nature01116

[21] Dornan, D., Shimizu, H., Burch, L., Smith, A.J. and Hupp, T.R. (2003) The proline repeat domain of p53 binds directly to the transcriptional coactivator p300 and allosterically controls DNA-dependent acetylation of p53. Molecular and Cellular Biology, 23, 8846-8861. doi:10.1128/MCB.23.23.8846-8861.2003

[22] Helton, E.S., Zhu, J. and Chen, X. (2006) The unique $\mathrm{NH}_{2}-$ terminally deleted $(\Delta \mathrm{N})$ residues, the PXXP motif, and the PPXY motif are required for the transcriptional activity of the $\Delta \mathrm{N}$ variant of p63. Journal of Biological Chemistry. 281, 2533-2542. doi:10.1074/jbc.M507964200

[23] Saner, K.J., Suzuki, T., Sasano, H., Pizzey, J., Ho, C., Strauss III, J.F., Carr, B.R., Rainey, W.E. (2005) Steroid sulfotransferase $2 \mathrm{~A} 1$ gene transcription is regulated by steroidogenic factor 1 and GATA-6 in the human adrenal. Molecular Endocrinology, 19, 184-197. doi:10.1210/me.2003-0332

[24] Brewer, A., Nemer, G., Gov, C., Rawlins, F., Nemer, M., Patient, R. and Pizzey, J. (2002) Widespread expression of an extended peptide sequence of GATA- 6 during murine embryogenesis and non-equivalence of RNA and protein expression domains. Mechanisms of Development, 119, S121-129. doi:10.1016/S0925-4773(03)00104-7

[25] Perlman, H., Suzuki, E., Simonson, M., Smith, R.C. and Walsh, K. (1998) GATA-6 induces p21 ${ }^{\mathrm{Cip} 1}$ expression and $\mathrm{G}_{1}$ cell cycle arrest. Journal of Biological Chemistry, 273, 13713-13718. doi:10.1074/jbc.273.22.13713

[26] Frankenberg, S., Gerbe, F., Bessonnard, S., Belville, C., Pouchin, P., Bardot, O. and Chazaud, C. (2011) Primitive endoderm differentiates via a three-step mechanism involving Nang and RTK signaling. Developmental Cell, 21, 1005-1013. doi:10.1016/j.devcel.2011.10.019 